\title{
Prevalência e fatores associados à constipação intestinal funcional em universitários
}

\author{
Prevalence and factors associated with functional constipation in university students
}

\author{
Edilene Vitorino Olivona, Juliana de Jesus Costa ${ }^{a}$, Alisson Diego Machado ${ }^{b}$, \\ Daniela Maria Alves Chaudc, Edeli Simioni de Abreu \\ Nutricionista graduada pela Universidade Presbiteriana Mackenzie. \\ Nutricionista. Mestrando em Nefrologia. Universidade de São Paulo. \\ c Nutricionista. Doutora em Pediatria e Ciências Aplicadas à Pediatria. Universidade Presbiteriana Mackenzie. \\ d Nutricionista. Doutora em Saúde Pública. Universidade Presbiteriana Mackenzie.
} privadas localizadas no município de São Paulo-SP. A coleta de dados foi realizada por meio do preenchimento de um questionário autoadministrado, contendo perguntas referentes à idade, sexo, etnia, prática de atividade física, tabagismo, peso e estatura autorreferidos, curso de graduação, história familiar de constipação, número de refeições diárias, ingestão de alimentos-fonte de fibras e ingestão hídrica. A presença de constipação intestinal funcional foi determinada de acordo com os critérios de Roma III. Foram criados modelos de regressão logística, em que foram estabelecidas as razões de prevalência bruta e ajustada de acordo com as variáveis em estudo.

Resultados: Foram avaliados 90 universitários (70 do sexo feminino), com média de idade de 26,8 8 8,6 anos. A prevalência de constipação intestinal funcional foi de 20,0\%. Observou-se que os sintomas mais prevalentes foram menos de três evacuações por semana (30,0\%), esforço evacuatório $(21,1 \%)$, fezes grumosas ou duras $(21,1 \%)$ e sensação de evacuação incompleta $(17,8 \%)$. No modelo multivariado, houve diferença estatística marginal apenas para o sexo, sendo que a prevalência foi maior no sexo masculino em relação ao feminino $(35,0 \%$ e $15,7 \%$, respectivamente, $p=0,06)$. Para os demais fatores, não se observou diferença estatística.

Conclusão: A prevalência de constipação intestinal funcional na amostra de estudo pode ser considerada elevada. Não foi identificada associação com fatores de risco clássicos para constipação.

Palavras-chave: constipação intestinal; prevalência; estudantes.

\section{ABSTRACT}

Objective: To evaluate the prevalence and factors associated with functional constipation in university students.

Materials and Methods: A cross-sectional study was performed with 90 university students from private higher education institutions located in São Paulo-SP. Data collection was performed by completing a self-administered questionnaire with questions about age, sex, ethnicity, practice of physical activity, tobacco use, self-reported weight and height, undergraduate course, family history of constipation, number of daily meals, fiber and water intake. Presence of functional constipation was determined according to Rome III criteria. Logistic regression models were created, in which establishes crude and adjusted hazard ratios according to study variables. Results: 90 university students (70 female) with a mean age of $26.8 \pm 8.6$ years old were evaluated. Prevalence of functional constipation was $20.0 \%$. The most prevalent symptoms were fewer than three evacuations per week (30.0\%), straining (21.1\%), lumpy or hard stools (21.1\%) and sensation of incomplete evacuation (17.8\%). In the multivariate model, there was marginal statistical difference only for sex, wherein the prevalence was higher in males compared to females $(35.0 \%$ and $15.7 \%$, respectively, $p=0.06)$. For other factors, there was no statistical difference.

Conclusion: Prevalence of functional constipation in the study sample can be considered elevated. No association was found with classic risk factors for constipation.

Keywords: constipation; prevalence; students.

\section{Correspondência:}




\section{INTRODUÇÃO}

A constipação intestinal é um sintoma caracterizado por frequência de evacuação inferior a 3 vezes por semana, fezes grumosas ou duras, esforço evacuatório e sensação de evacuação incompleta, por pelo menos seis meses ${ }^{1}$. Embora a constipação intestinal não apresente um risco à vida, pode causar desconforto e impactar significativamente a qualidade de vida ${ }^{2}$.

A constipação intestinal pode ser classificada quanto à sua origem, em orgânica ou funcional. A constipação intestinal orgânica é decorrente de alguma condição metabólica ou doença, como hipo e hipercalemia, hipotireoidismo severo, diabetes mellitus, doença de Parkinson, esclerose múltipla e doenças que afetem a região colorretal, bem como pela utilização de medicamentos, como anticolinérgicos, bloqueadores de canais de cálcio e antiácidos. Por sua vez, a constipação intestinal funcional se caracteriza pela ausência de causas orgânicas definidas e está relacionada ao estilo de vida, decorrente de uma dieta pouco saudável, sedentarismo e estresse ${ }^{3}$.

O diagnóstico da constipação intestinal geralmente é feito por meio dos critérios de Roma $\mathrm{II}^{4}$, que utiliza como parâmetros a presença de esforço evacuatório, fezes grumosas ou duras, sensação de evacuação incompleta, sensação de obstrução ou bloqueio anorretal das fezes, manobras manuais para facilitar a evacuação, menos de três evacuações por semana e escape fecal sem a utilização de laxantes, sendo que a apresentação de dois ou mais sintomas nos últimos três meses e com início há pelo menos seis meses sugere o diagnóstico de constipação intestinal.

A prevalência de constipação intestinal funcional na população brasileira chega a $36,8 \%$, sendo maior no sexo feminino, em idosos, em sedentários e em pessoas com baixa ingestão hídrica e de fibras e de menor nível socioeconômico ${ }^{5,6,7,8}$. Os universitários também representam um grupo de risco para o desenvolvimento de constipação intestinal funcional, uma vez que os mesmos apresentam nesse momento de suas vidas uma maior responsabilidade sobre si, somados a fatores psicossociais e ao ambiente universitário, como hábitos alimentares inadequados e sedentarismo ${ }^{9}$.

Assim, o presente estudo teve por objetivo avaliar a prevalência e os fatores associados à constipação intestinal funcional em universitários.

\section{MATERIAIS E MÉTODOS}

Foi realizado um estudo transversal com uma amostra de conveniência, composta por 90 universitários de instituições de ensino superior privadas localizadas no município de São
Paulo-SP. Foi utilizado como critério de inclusão idade igual ou superior a 18 anos.

A coleta de dados foi realizada em 2014, por meio do preenchimento de um questionário autoadministrado, contendo perguntas referentes à idade, sexo, etnia, prática de atividade física, tabagismo, peso autorreferido, estatura autorreferida, curso de graduação, história familiar de constipação, número de refeições diárias, ingestão de alimentos-fonte de fibras e ingestão hídrica. Considerou-se praticante de atividade física o universitário que relatou a prática em pelo menos 3 vezes por semana, tanto de exercícios aeróbicos quanto anaeróbicos, com duração mínima de 30 minutos/dia. Para a ingestão de alimentos-fonte de fibras, o estudante deveria assinalar se consumia diariamente ou não e a quantidade de porções, sendo que uma quantidade igual ou superior a 3 porções/dia foi considerada como ingestão diária desse nutriente, uma vez que fornece cerca de 25 gramas/dia de fibras, conforme indicado no Guia alimentar para a população brasileira ${ }^{10}$. As porções foram definidas de acordo com as sugeridas pelo Guia.

Para a classificação do estado nutricional calculou-se o índice de massa corporal (IMC), em que IMC $<16,0 \mathrm{~kg} / \mathrm{m}^{2}$ indica magreza grau III; $16,0-16,9 \mathrm{~kg} / \mathrm{m}^{2}$, magreza grau II; $17,0-18,4 \mathrm{~kg} / \mathrm{m}^{2}$, magreza grau I; $18,5-24,9 \mathrm{~kg} / \mathrm{m}^{2}$, eutrofia; $25,0-29,9 \mathrm{~kg} / \mathrm{m}^{2}$, sobrepeso; $30,0-34,5 \mathrm{~kg} / \mathrm{m}^{2}$, obesidade grau I; 35,0-39,9 kg/m²; e $\geq 40,0 \mathrm{~kg} / \mathrm{m}^{2}$, obesidade grau III'11.

A presença de constipação intestinal funcional foi determinada de acordo com os critérios de Roma III $^{4}$. Foram considerados constipados os universitários que apresentaram dois ou mais dos seguintes parâmetros, sendo preenchidos nos últimos três meses e com início dos sintomas pelo menos seis meses antes do diagnóstico: esforço evacuatório durante pelo menos $25 \%$ das evacuações, fezes grumosas ou duras em pelo menos $25 \%$ das evacuações, sensação de evacuação incompleta em pelo menos $25 \%$ das evacuações, sensação de obstrução ou bloqueio anorretal das fezes em pelo menos $25 \%$ das evacuações, manobras manuais para facilitar a evacuação em pelo menos $25 \%$ das mesmas, menos de três evacuações por semana e presença de escape fecal sem a utilização de laxantes.

Os dados foram apresentados em frequências absoluta e relativa. Para a verificação da diferença da prevalência de constipação intestinal funcional, as variáveis foram dicotomizadas e foram criados modelos de regressão logística, em que foram estabelecidas as razões de prevalência bruta e ajustada, por idade e sexo. Foi aplicado o teste exato de Fisher para a verificação da diferença entre variáveis quantitativas. Foi considerada diferença significativamente estatística $p<0,05$. As análises estatísticas foram realizadas no software SPSS versão 17.0. 
A coleta de dados foi realizada após a assinatura do Termo de Consentimento Livre e Esclarecido por todos os participantes do estudo. Foram seguidas as determinações da Resolução no 466/12, do Conselho Nacional de Saúde. O estudo foi aprovado pelo Comitê Interno de Ética em Pesquisa da Universidade Presbiteriana Mackenzie, sob número N005/03/13.

\section{RESULTADOS}

Foram avaliados 90 estudantes universitários (70 do sexo feminino), com média de idade de $26,8 \pm 8,6$ anos. A maioria da amostra era de etnia branca, sedentária, não-tabagista, eutrófica, fazendo algum curso da área de biológicas e que não possuíam antecedentes de constipação na família. Em relação aos hábitos alimentares, a maioria realizava 4 refeições ou mais por dia e não ingeriam alimentos-fonte de fibras diariamente, bem como consumiam 4 copos ou mais de líquidos por dia (Tabela 1).

A prevalência de constipação intestinal funcional foi de 20,0\%. Observou-se, em relação aos critérios de Roma III, que os sintomas mais prevalentes foram menos de três evacuações por semana (30,0\%), seguido de esforço evacuatório $(21,1 \%)$, fezes grumosas ou duras $(21,1 \%)$ e sensação de evacuação incompleta $(17,8 \%)$. Nenhum dos participantes relatou a necessidade de manobras manuais para facilitar a evacuação, a presença de escape fecal sem a utilização de laxantes e a sensação de obstrução ou bloqueio anorretal das fezes, conforme demonstrado na Tabela 2. Embora nenhum dos participantes tenha relatado realizar algum tipo de manobra manual para facilitar a evacuação, $6,7 \%(n=6)$ dos participantes relataram o uso de algum laxativo.

No modelo multivariado, houve diferença estatística marginal apenas para a variável sexo, sendo que a prevalência foi maior no sexo masculino, em que o sexo feminino apresentou uma prevalência $66 \%$ menor (Tabela 3). Para os demais fatores, não observou-se diferença estatística.

\section{DISCUSSÃO}

A prevalência de constipação intestinal funcional na amostra em estudo correspondeu a 20,0\%, havendo diferença estatística marginal apenas para o sexo, sendo que a prevalência foi maior no sexo masculino.

Em estudo realizado por Jaime et al. ${ }^{12}$, foi identificada uma prevalência de constipação intestinal de 40,0\% em 200 universitários de uma instituição de ensino superior localizada em Goiânia-GO, sendo a prevalência maior em estudantes com história familiar de constipação. Trisóglio et al. ${ }^{13}$, ao realizarem um estudo com 150 estudantes de Medicina de uma instituição no noroeste paulista, verificaram
Tabela 1. Caracterização de uma amostra de universitários.

\begin{tabular}{|c|c|c|}
\hline Variável & $\mathbf{n}$ & $\%$ \\
\hline \multicolumn{3}{|l|}{ Idade } \\
\hline$<23$ anos & 42 & 46,7 \\
\hline$\geq 23$ anos & 48 & 53,3 \\
\hline \multicolumn{3}{|l|}{ Sexo } \\
\hline Masculino & 20 & 22,2 \\
\hline Feminino & 70 & 77,8 \\
\hline \multicolumn{3}{|l|}{ Etnia } \\
\hline Não-branca & 25 & 27,8 \\
\hline Branca & 65 & 72,2 \\
\hline \multicolumn{3}{|l|}{ Prática de atividade física } \\
\hline Sim & 44 & 48,9 \\
\hline Não & 46 & 51,1 \\
\hline \multicolumn{3}{|l|}{ Tabagismo } \\
\hline Sim & 10 & 11,1 \\
\hline Não & 80 & 88,9 \\
\hline \multicolumn{3}{|l|}{ Excesso de peso } \\
\hline Sim & 31 & 34,4 \\
\hline Não & 59 & 65,6 \\
\hline \multicolumn{3}{|l|}{ Curso } \\
\hline Biológicas & 59 & 65,6 \\
\hline Outras áreas & 31 & 34,4 \\
\hline \multicolumn{3}{|l|}{ Constipação na família } \\
\hline Sim & 43 & 47,8 \\
\hline Não & 47 & 52,2 \\
\hline \multicolumn{3}{|l|}{ Número de refeições/dia } \\
\hline$<4$ & 24 & 26,7 \\
\hline$\geq 4$ & 66 & 73,3 \\
\hline \multicolumn{3}{|c|}{ Ingestão diária de alimentos-fonte de fibras } \\
\hline $\operatorname{Sim}$ & 43 & 47,8 \\
\hline Não & 47 & 52,2 \\
\hline \multicolumn{3}{|l|}{ Ingestão hídrica } \\
\hline$<4$ copos/dia & 30 & 33,3 \\
\hline$\geq 4$ copos/dia & 60 & 66,7 \\
\hline Total & 90 & 100,0 \\
\hline
\end{tabular}

Tabela 2. Prevalência dos sintomas de constipação intestinal funcional em universitários.

\begin{tabular}{lcc}
\hline \multicolumn{1}{c}{ Critério } & n & $\%$ \\
\hline Menos de três evacuações por semana & 27 & 30,0 \\
Esforço evacuatório* & 19 & 21,1 \\
Fezes grumosas ou duras* & 19 & 21,1 \\
Sensação de evacuação incompleta* & 16 & 17,8 \\
Manobras manuais para facilitar a evacuação* & - & - \\
$\begin{array}{l}\text { Presença de escape fecal sem a utilização de } \\
\text { laxantes }\end{array}$ & - & - \\
$\begin{array}{l}\text { Sensação de obstrução ou bloqueio anorretal } \\
\text { das fezes* }\end{array}$ & - & - \\
\hline
\end{tabular}

* Em pelo menos $25 \%$ das defecações. 
Tabela 3. Prevalência e razões de prevalência bruta e ajustada de constipação intestinal funcional em universitários de acordo com variáveis de estudo.

\begin{tabular}{|c|c|c|c|c|c|}
\hline Variável & $\begin{array}{c}\text { Prevalência } \\
(\%)\end{array}$ & $\begin{array}{l}\text { RP bruta } \\
\text { (IC95\%) }\end{array}$ & $\mathbf{p}$ & $\begin{array}{l}\text { RP ajustada } \\
\text { (IC95\%) }\end{array}$ & $\mathbf{p}$ \\
\hline Idade & & & 0,82 & & \\
\hline$<23$ anos & 16,7 & $\begin{array}{c}0,86 \\
(0,24-3,14)\end{array}$ & & & \\
\hline$\geq 23$ anos & 22,9 & 1 & & & \\
\hline Sexo & & & 0,08 & & 0,06 \\
\hline Masculino & 35,0 & 1 & & 1 & \\
\hline Feminino & 15,7 & $\begin{array}{c}0,22 \\
(0,04-1,18)\end{array}$ & & $\begin{array}{c}0,34 \\
(0,11-1,07)^{a}\end{array}$ & \\
\hline Etnia & & & 0,30 & & \\
\hline Não-branca & 12,0 & 1 & & & \\
\hline Branca & 23,1 & $\begin{array}{c}2,24 \\
(0,48-10,34)\end{array}$ & & & \\
\hline Prática de atividade física & & & 0,16 & & \\
\hline Sim & 15,9 & 1 & & & \\
\hline Não & 23,9 & $\begin{array}{c}2,54 \\
(0,70-9,25)\end{array}$ & & & \\
\hline Tabagismo & & & 0,69 & & \\
\hline Sim & 20,0 & $\begin{array}{c}1,43 \\
(0,25-8,06)\end{array}$ & & & \\
\hline Não & 20,0 & 1 & & & \\
\hline Excesso de peso & & & 0,82 & & \\
\hline $\operatorname{Sim}$ & 25,8 & $\begin{array}{c}1,19 \\
(0,27-5,13)\end{array}$ & & & \\
\hline Não & 16,9 & 1 & & & \\
\hline Curso & & & 0,36 & & \\
\hline Biológicas & 16,9 & 1 & & & \\
\hline Outras áreas & 25,8 & $\begin{array}{c}2,30 \\
(0,39-13,45)\end{array}$ & & & \\
\hline Constipação na família & & & 0,36 & & \\
\hline Sim & 23,3 & $\begin{array}{c}1,80 \\
(0,51-6,44)\end{array}$ & & & \\
\hline Não & 17,0 & 1 & & & \\
\hline Número de refeições/dia & & & 0,07 & & 0,11 \\
\hline$<4$ & 33,3 & $\begin{array}{c}3,52 \\
(0,90-13,78)\end{array}$ & & $\begin{array}{c}2,49 \\
(0,82-7,58)^{b}\end{array}$ & \\
\hline$\geq 4$ & 15,2 & 1 & & 1 & \\
\hline Ingestão diária de alimentos - fonte de fibras & & & 0,95 & & \\
\hline Sim & 20,9 & 1 & & & \\
\hline Não & 19,1 & $\begin{array}{c}0,96 \\
(0,29-3,23)\end{array}$ & & & \\
\hline Ingestão hídrica & & & 0,38 & & \\
\hline$<4$ copos/dia & 26,7 & $\begin{array}{c}1,75 \\
(0,50-6,14)\end{array}$ & & & \\
\hline$\geq 4 \mathrm{copos} /$ dia & 16,7 & 1 & & & \\
\hline
\end{tabular}

a Ajustada por idade; ${ }^{b}$ Ajustada por idade e sexo.

As análises ajustadas contêm as variáveis com $\mathrm{p}<0,20$.

uma prevalência de constipação intestinal de 35,0\%, sendo maior no sexo feminino. Por sua vez, Braz e Lessa ${ }^{14}$ identificaram uma prevalência de constipação intestinal de 22,5\% em 40 universitários de Nutrição de um centro universitário localizado em Minas Gerais.
A prevalência de constipação intestinal funcional foi menor no presente estudo em relação aos demais, com exceção de Braz e Lessa ${ }^{14}$, que verificaram uma prevalência semelhante. Ainda, a prevalência foi maior no sexo masculino, ao contrário do verificado por Trisóglio 
et al. $^{13}$ e por outros estudos realizados na população em geral ${ }^{5,6,7}$.

No presente estudo, possivelmente a prevalência de constipação intestinal funcional tenha sido menor em relação a outros estudos devido a $60,0 \%(n=54)$ da amostra ser composta por graduandos de Nutrição, que possuem um maior conhecimento em relação ao tema. Além disso, a amostra de estudo é formada por estudantes de classes socioeconômicas elevadas, em que a prevalência de constipação intestinal é menor ${ }^{5}$.

Quanto à maior prevalência de constipação intestinal no sexo masculino, tal fato pode ter sido decorrente de um possível viés de seleção, uma vez que no presente estudo há uma maior proporção de estudantes do sexo masculino na área de exatas ou humanas do que na de biológicas $(51,6 \%$ e $6,8 \%$, respectivamente, $p<0,001$ ), podendo se preocupar menos com aspectos relacionados à saúde.

Em relação aos sintomas, embora nenhum dos participantes tenha relatado realizar algum tipo de manobra manual para facilitar a evacuação, 6,7\% $(n=6)$ utilizavam algum laxativo. $\mathrm{O}$ uso de laxativos é uma manobra muito empregada por estarem facilmente disponíveis. Os mais diversos tipos de laxantes compartilham a capacidade de reduzir a consistência das fezes e/ou aumentar os movimentos peristálticos. No entanto, provocam irritação e inflamação na mucosa gastrointestinal, ao mesmo tempo em que estimulam evacuação completa em curto prazo, de modo dose-dependente. A interrupção da sua utilização está associada à constipação, levando à retomada ao uso e criando assim um ciclo vicioso ${ }^{15}$.

Embora no presente estudo não tenha sido verificada associação entre a presença de constipação intestinal funcional e o relato de ingestão de alimentos-fonte de fibras, já foi demonstrada uma relação inversa ${ }^{16}$, assim como a ingestão hídrica ${ }^{17}$. Além disso, a utilização de fibras demonstrou ser eficaz no tratamento da constipação ${ }^{18,19,20}$, bem como o de probióticos ${ }^{21,22,23}$.

Programas que estimulem um maior cuidado com a saúde, tendo em vista a promoção da melhora dos hábitos alimentares, da prática de atividade física e do estilo de vida podem ser benéficos nesta população.

Como fatores limitantes do estudo, destacam-se o viés de seleção da amostra e o fato da mesma ser homogênea, composta em grande parte por adultos jovens e de classes socioeconômicas elevadas. Além disso, alguns dados foram autorreferidos, fazendo com que eventuais falhas nos relatos possam enviesar os achados do estudo.

Assim, verificou-se que a prevalência de constipação intestinal funcional em universitários de instituições de ensino superior pode ser considerada alta e, no presente estudo, maior no sexo masculino, não estando associada a fatores de risco clássicos. Ainda, ressalta-se a importância do manejo de fatores de risco modificáveis para esse sintoma, com uma ingestão de fibras e hídrica adequada, a prática de atividade física e a adoção de um estilo de vida saudável.

\section{REFERÊNCIAS}

1. Paré $P$, Bridges R, Champion MC, Ganguli SC, Gray JR, Irvine EJ, Plourde V, Poitras P, Turnbull GK, Moayyedi P, Flook N, Collins SM. Recommendations on chronic constipation (including constipation associated with irritable bowel syndrome) treatment. Can J Gastroenterol. 2007;21:3B-22B. http://dx.doi. org/10.1155/2007/848706

2. Friedenberg FK, Dadabhai A, Palit A, Sankineni A. The impact of functional constipation on quality of life of middle-aged black Americans: a prospective case-control study. Qual Life Res. 2012;21:1713-7. http://dx.doi.org/10.1007/s11136-011-0089-z

3. Gray JR. What is chronic constipation? Definition and diagnosis. Can J Gastroenterol. 2011;25:7B-10B. http://dx.doi. org/10.1155/2011/548024

4. Longstreth GF, Thompson WG, Chey WD, Houghton LA, Mearin F, Spiller RC. Functional bowel disorders. Gastroenterology. 2006;130:1480-91. http://dx.doi.org/10.1053/j.gastro.2005.11.061

5. Collete VL, Araújo CL, Madruga SW. Prevalência e fatores associados à constipação intestinal: um estudo de base populacional em Pelotas, Rio Grande do Sul, Brasil, 2007. Cad Saúde Pública. 2010;26:13911402. http://dx.doi.org/10.1590/S0102-311X2010000700018

6. Klaus JH, Nardin V, Paludo J, Scherer F, Dal Bosco SM. Prevalência e fatores associados à constipação intestinal em idosos residentes em instituições de longa permanência. Rev Bras Geriatr Gerontol. 2015;18:835-43. http://dx.doi.org/10.1590/18099823.2015.13175

7. Schmidt FMQ, Santos VLCG, Domansky RC, Barros E, Bandeira MA, Tenório MAM, Jorge JMN. Prevalência de constipação intestinal autorreferida em adultos da população geral. Rev Esc Enferm USP. 2015;49:443-52. http://dx.doi.org/1590/S0080623420150000300012

8. Sacomori C, Silveira C, Sperandio FF, Cardoso FL. Prevalence, repercussion and factors associated with intestinal constipation in women in Florianópolis. J Coloproctol. 2014;34:254-9. http:// dx.doi.org/10.1016/j.jcol.2014.08.006

9. Dong YY, Chen FX, Yu YB, Du C, Qi QQ, Liu H, Li YQ. A schoolbased study with Rome III criteria on the prevalence of functional gastrointestinal disorders in Chinese college and university students. PLoS One. 2013;8:e54183. http://dx.doi.org/10.1371/journal. pone.0054183

10. Ministério da Saúde (BR). Secretaria de Atenção à Saúde. Departamento de Atenção Básica. Guia alimentar para a população brasileira: promovendo a alimentação saudável. Brasília: Ministério da Saúde; 2008.

11. World Health Organization. Obesity: preventing and managing the global epidemic. Geneva: WHO; 2000.

12. Jaime RP, Campos RC, Santos TST, Marques MS. Prevalência e fatores de risco da constipação intestinal em universitários de uma instituição particular de Goiânia, GO. Rev Inst Ciênc Saúde. 2009;27:378-83. 
13. Trisóglio C, Marchi CMG, Torres US, Netinho JG. Prevalência de constipação intestinal entre estudantes de medicina de uma instituição no Noroeste Paulista. Rev Bras Colo-Proctol. 2010;30: 203-9. http://dx.doi.org/10.1590/S0101-98802010000200012

14. Braz CRS, Lessa NMV. Fatores de risco e prevalência de constipação intestinal em graduandos do curso de Nutrição de um centro universitário de Minas Gerais. Nutrir Gerais. 2011;5:740-54.

15. Billodre NB, Gomez R, Caletti G. Prevalência e motivação de uso de laxantes entre universitárias do curso de nutrição do Centro Universitário Metodista de Porto Alegre/RS. Ciênc Mov. 2013;15:3543. http://dx.doi.org/10.15602/1983-9480/cmbs.v15n30p35-43

16. Khatri PK, Ali AD, Alzadjali N, Bhagia G, Khaligdina SJ, Aziz S. Frequency of functional constipation in 3 different populations and its causative factors. J Pak Med Assoc. 2011;61:1149-52.

17. Markland AD, Palsson O, Goode PS, Burgio KL, Busby-Whitehead J, Whitehead WE. Association of low dietary intake of fiber and liquids with constipation: evidence from the National Health and Nutrition Examination Survey (NHANES). Am J Gastroenterol. 2013;108: 796-803. http://dx.doi.org/10.1038/ajg.2013.73

18. Machado WM, Capelari SM. Avaliação da eficácia e do grau de adesão ao uso prolongado de fibra dietética no tratamento da constipação intestinal funcional. Rev Nutr. 2010;23:231-8. http:// dx.doi.org/10.1590/S1415-52732010000200006

19. Woo HI, Kwak SH, Lee Y, Choi JH, Cho YM, Om AS. A controlled, randomized, double-blind trial to evaluate the effect of vegetables and whole grain powder that is rich in dietary fibers on bowel functions and defecation in constipated young adults. J Cancer Prev. 2015;20:64-9. http://dx.doi.org/10.15430/JCP.2015.20.1.64

20. Cong L, Ma JT, Jin ZJ, Duan LW, Su WP, Zheng J, Zhang LJ, Xu J, Li DF. Efficacy and safety of high specific volume polusaccharide-A new type of dietary fiber for treatment of functional constipation and IBS-C. J Nutr Sci Vitaminol. 2015;61:326-31. http://dx.doi. org/10.3177/jnsv.61.326

21. Riezzo G, Orlando A, D'Attoma B, Guerra V, Valerio F, Lavermicocca P, De Candia S, Russo F. Randomised clinical trial: efficacy of Lactobacillus paracasei-enriched artichokes in the treatment of patients with functional constipation - a double-blind, controlled, crossover-study. Aliment Pharmacol Ther. 2012;35:441-50. http:// dx.doi.org/10.1111/j.1365-2036.2011.04970.x

22. Favretto DC, Pontin B, Moreira TR. Effect of the consumption of a cheese enriched with probiotic organisms (Bifidobacterium lactis Bi-07) in improving symptoms of constipation. Arq Gastroenterol. 2013;50:196-201. http://dx.doi.org/10.1590/S000428032013000200035

23. Magro DO, Oliveira LMR, Bernasconi I, Ruela MS, Credidio L, Barcelos IK, Leal RF, Ayrizono MLS, Fagundes JJ, Teixeira LB, Ouwehand AC, Coy CSR. Effect of yogurt containing polydextrose, Lactobacillus acidophilus NCFM and Bifidobacterium lactis HN019: a randomized, double-blind, controlled study in chronic constipation. Nutr J. 2014;13:75. http://dx.doi.org/10.1186/14752891-13-75 